\title{
20. SEISMIC VELOCITIES, DENSITIES, AND POROSITIES OF LAYER 2B AND LAYER 2C BASALTS FROM HOLE 504B 1
}

\author{
Nikolas I. Christensen, Department of Geosciences, Purdue University \\ and \\ Matthew H. Salisbury, Scripps Institution of Oceanography²
}

\begin{abstract}
Compressional and shear wave velocities at confining pressures to $6 \mathrm{~kb}$, densities, and porosities were measured for 32 samples obtained from 836 to $1350 \mathrm{~m}$ below seafloor (BSF) in Hole 504B, the section drilled on Leg 83 of the Deep Sea Drilling Project. These data in combination with similar measurements on 28 basalt samples from the section from 274.5 to $836 \mathrm{~m}$, drilled on Legs 69 and 70 , provide a comprehensive set of physical property data for over $1000 \mathrm{~m}$ of oceanic crust. The velocities, densities, and porosities measured in the laboratory exhibit greater variability in the upper portion of the hole. In general, compressional and shear wave velocities and densities increase with depth, reaching average values at $1 \mathrm{kbar}$ of $V_{\mathrm{p}}=6.45 \mathrm{~km} / \mathrm{s}, V_{\mathrm{s}}=3.45 \mathrm{~km} / \mathrm{s}$ and $\rho=2.94 \mathrm{~g} / \mathrm{cm}^{3}$ within the sheeted dike section. Porosities decrease with depth to values generally less than $1 \%$ near the bottom of the hole.
\end{abstract}

\section{INTRODUCTION}

Drilling during Legs 69, 70, and 83 of the Deep Sea Drilling Project has provided samples of the oceanic crust to a depth of $1350 \mathrm{~m}$ below the seafloor (BSF) and 1075 $\mathrm{m}$ into basement. The bottom $514 \mathrm{~m}$, cored during Leg 83 , is characterized by a transition from pillow lavas, pillow breccias, hyaloclastites, and basalt flows to an underlying unit interpreted as a sheeted dike complex similar to that observed in many ophiolites. Geophysical logging and other downhole experiments have found significant physical property changes with depth (Anderson, et al., 1982; Becker et al., 1982).

Laboratory data on the physical properties of the rocks recovered by the drilling are essential to the interpretation of the downhole geophysical measurements. For example, laboratory measurements of seismic velocities, porosities, and densities under appropriate conditions of confining pressure and water saturation provide information about the relative importance of porosity and alteration for the in situ properties. This paper reports compressional and shear wave velocities, densities, porosities, Poisson's ratios, shear moduli, and bulk moduli for 32 samples obtained from the lower $514 \mathrm{~m}$ of Hole 504B. These data, combined with similar results for basalt samples from the upper portion of the hole (Wilkens et al., 1983), provide a complete set of laboratory velocities and densities for interpretation of the logging data.

\section{EXPERIMENTAL PROCEDURE AND DATA}

The bulk densities of cylindrical water-saturated samples (Table 1) were determined from the dimensions of the samples and their weight in air. All samples were stored in water at the time of drilling. After the velocity runs, the samples were air dried for several weeks and re-

\footnotetext{
${ }^{1}$ Anderson, R. N., Honnorez, J., Becker, K., et al., Init. Repts. DSDP, 83: Washington (U.S. Govt. Printing Office).

2 Addresses: (Christensen) Department of Geosciences, Purdue University, West Lafayette, IN 47907; (Salisbury, present address) Centre for Marine Geology, Dalhousie University, Halifax, Nova Scotia, Canada B3H 3 J5.
}

weighed for calculation of dry bulk densities. The porosities in Table 1 were calculated from the wet and dry densities.

Compressional and shear wave velocities were measured using the pulse transmission technique described in detail by Birch (1960). All velocities were measured at room temperature using water-saturated samples. The samples were jacketed with copper foil and 100-mesh screens were placed between the cores and copper jackets to provide space for water to drain from microcracks as the confining pressure was increased. Thus, pore pressure was kept much lower than confining pressure during the pressure cycles.

In addition to wet bulk densities $(\rho)$ and porosities $(\phi)$, compressional and shear wave velocities $\left(V_{\mathrm{p}}\right.$ and $\left.V_{\mathrm{s}}\right)$, velocity ratios $\left(V_{\mathrm{p}} / V_{\mathrm{s}}\right)$, Poisson's ratio $(\sigma)$, bulk moduli $(K)$ and shear moduli $(\mu)$ are presented in Table 1. The elastic constants were calculated from the velocities and densities using the equations summarized by Birch (1961).

\section{DISCUSSION}

Comparisons of the laboratory data with similar logging and field measurements provide important constraints on large-scale porosity within the crust. It is concluded from such comparisons that the fracture porosity is greatest near the top of Layer 2, decreases with depth, and approaches zero near the bottom $100 \mathrm{~m}$ of the hole (Becker et al., 1982; Becker, this volume; Salisbury et al., this volume).

There also exists a significant, systematic change in velocities and densities with depth on a smaller scale. This is shown in Table 2 where the average laboratory velocities at $1 \mathrm{~kb}$, porosities, and densities are tabulated at selected depth intervals. Compressional and shear wave velocities, as well as densities, show a systematic increase with depth. Much of this change is attributed to a decrease in microcracks and micropores with depth, since the velocities and densities show an excellent correlation with the measured porosities of the cores (Table 2).

The properties measured in the laboratory for the lowermost samples of Hole 504B are of special interest since the samples represent the first obtained by drilling into seismic Layer $2 \mathrm{C}$. Using the thicknesses and densities of the overlying seawater, sediment, and basalt columns, the 
confining pressure at the base of Hole 504B is calculated to be $0.7 \mathrm{~kb}$. If at crustal depths equivalent to the base of the hole the rock pore spaces are connected to the seawater column, which is likely at least in the vicinity of the drill hole (Salisbury et al., this volume), the pore pressure will be $0.49 \mathrm{~kb}$. If velocity is simply a function of differential pressure (confining pressure minus pore pressure), laboratory velocities at $0.2 \mathrm{~kb}$ should be approximately representative of in situ conditions at the base of Hole 504B. Average laboratory compressional and shear wave velocities for the lower five samples are 6.27 and $3.29 \mathrm{~km} / \mathrm{s}$ at $0.2 \mathrm{~kb}$ (Table 1).

\section{ACKNOWLEDGMENTS}

Financial support was provided by the Office of Naval Research Contract N-00014-80-C-0252.

\section{REFERENCES}

Anderson, R. N., Honnorez, J., Becker, K., Adamson, A. C., Alt, J. C., Emmermann, R., Kempton, P. D., Kinoshita, H., Laverne, C.,
Mottl, M. J., and Newmark, R. L., 1982. DSDP Hole 504B, the first reference section over $1 \mathrm{~km}$ through Layer 2 of the oceanic crust. Nature, 300:589-594.

Becker, K., Von Herzen, R. P., Francis, T. J. G., Anderson, R. N., Honnorez, J., Adamson, A. C., Alt, J. C., Emmermann, R., Kempton, P. D., Kinoshita, H., Laverne, C., Mottl, M. J., and Newmark, R. L., 1982. In situ electrical resistivity and bulk porosity of the oceanic crust Costa Rica Rift. Nature, 300:594-598.

Birch, F., 1960. The velocity of compressional waves in rocks to 10 kbar, 1. J. Geophys. Res., 65:1083-1102.

Birch, F., 1961. The velocity of compressional waves in rocks to 10 kbar, 2. J. Geophys. Res., 66:2199-2224.

Wilkens, R. H., Christensen, N. I., and Slater, L., 1983. High-pressure seismic studies of Leg 69 and 70 basalts. In Cann, J. R., Langseth, M. G., Honnorez, J., Von Herzen, R. P., White, S. M., et al., Init. Repts. DSDP, 69: Washington (U.S. Govt. Printing Office), 683-686.

Date of Acceptance: 12 March 1984 
Table 1. Compressional $\left(V_{\mathrm{p}}\right)$ and shear $\left(V_{\mathrm{S}}\right)$ wave velocities and elastic constants of Hole 504B basalts.

\begin{tabular}{|c|c|c|c|c|c|c|c|}
\hline $\begin{array}{c}\text { Sample } \\
\text { (interval in } \mathrm{cm} \text { ) }\end{array}$ & $\begin{array}{c}P \\
(\mathbf{k b})\end{array}$ & $\begin{array}{c}V_{\mathrm{p}} \\
(\mathrm{km} / \mathrm{s})\end{array}$ & $\begin{array}{c}V_{\mathrm{s}} \\
(\mathrm{km} / \mathrm{s})\end{array}$ & $V_{\mathrm{p}} / V_{\mathrm{s}}$ & $\sigma$ & $\begin{array}{c}K \\
(\mathrm{Mb})\end{array}$ & $\stackrel{\mu}{(M b)}$ \\
\hline \multirow{8}{*}{$\begin{aligned} & 504 \mathrm{~B}-72-2,33-35 \mathrm{~cm} \\
& \rho=2.87 \mathrm{~g} / \mathrm{cm}^{3} \\
& \phi=0.3 \%\end{aligned}$} & 0.2 & 6.30 & 3.30 & 1.91 & 0.31 & 0.72 & 0.31 \\
\hline & 0.4 & 6.34 & 3.33 & 1.90 & 0.31 & 0.73 & 0.32 \\
\hline & 0.6 & 6.33 & 3.34 & 1.89 & 0.31 & 0.72 & 0.32 \\
\hline & 0.8 & 6.38 & 3.35 & 1.90 & 0.31 & 0.74 & 0.32 \\
\hline & 1.0 & 6.39 & 3.36 & 1.90 & 0.31 & 0.74 & 0.32 \\
\hline & 2.0 & 6.43 & 3.37 & 1.91 & 0.31 & 0.76 & 0.33 \\
\hline & 4.0 & 6.47 & 3.39 & 1.91 & 0.31 & 0.77 & 0.33 \\
\hline & 6.0 & 6.50 & 3.39 & 1.92 & 0.31 & 0.78 & 0.33 \\
\hline \multirow{8}{*}{$\begin{aligned} 504 \mathrm{~B}-73-1,73-75 \mathrm{~cm} \\
\rho=2.91 \mathrm{~g} / \mathrm{cm}^{3} \\
\phi=0.3 \%\end{aligned}$} & 0.2 & 6.03 & 3.19 & 1.89 & 0.31 & 0.66 & 0.30 \\
\hline & 0.4 & 6.07 & 3.24 & 1.87 & 0.30 & 0.66 & 0.31 \\
\hline & 0.6 & 6.09 & 3.27 & 1.86 & 0.30 & 0.67 & 0.31 \\
\hline & 0.8 & 6.11 & 3.29 & 1.85 & 0.30 & 0.67 & 0.32 \\
\hline & 1.0 & 6.12 & 3.31 & 1.85 & 0.29 & 0.67 & 0.32 \\
\hline & 2.0 & 6.19 & 3.36 & 1.84 & 0.29 & 0.68 & 0.33 \\
\hline & 4.0 & 6.32 & 3.42 & 1.85 & 0.29 & 0.71 & 0.34 \\
\hline & 6.0 & 6.42 & 3.47 & 1.85 & 0.29 & 0.74 & 0.35 \\
\hline 504B-75-1, $13-15 \mathrm{~cm}$ & 0.2 & 5.70 & 2.93 & 1.94 & 0.32 & 0.59 & 0.24 \\
\hline$\rho=2.83 \mathrm{~g} / \mathrm{cm}^{3}$ & 0.4 & 5.74 & 2.96 & 1.93 & 0.32 & 0.61 & 0.25 \\
\hline$\phi=0.9 \%$ & 0.6 & 5.77 & 2.98 & 1.93 & 0.32 & 0.61 & 0.25 \\
\hline & 0.8 & 5.79 & 3.00 & 1.93 & 0.32 & 0.61 & 0.26 \\
\hline & 1.0 & 5.80 & 3.02 & 1.93 & 0.32 & 0.61 & 0.26 \\
\hline & 2.0 & 5.86 & 3.06 & 1.91 & 0.31 & 0.62 & 0.27 \\
\hline & 4.0 & 5.92 & 3.11 & 1.91 & 0.31 & 0.63 & 0.27 \\
\hline & 6.0 & 5.95 & 3.15 & 1.89 & 0.31 & 0.63 & 0.28 \\
\hline $504 \mathrm{~B}-77-3,5-7 \mathrm{~cm}$ & 0.2 & 5.84 & 3.13 & 1.87 & 0.30 & 0.61 & 0.28 \\
\hline$\rho=2.91 \mathrm{~g} / \mathrm{cm}^{3}$ & 0.4 & 5.89 & 3.19 & 1.84 & 0.29 & 0.61 & 0.30 \\
\hline$\phi=3.2 \%$ & 0.6 & 5.92 & 3.23 & 1.83 & 0.29 & 0.62 & 0.30 \\
\hline & 0.8 & 5.95 & 3.26 & 1.82 & 0.29 & 0.62 & 0.31 \\
\hline & 1.0 & 5.97 & 3.28 & 1.82 & 0.28 & 0.62 & 0.31 \\
\hline & 2.0 & 6.05 & 3.34 & 1.81 & 0.28 & 0.63 & 0.33 \\
\hline & 4.0 & 6.10 & 3.42 & 1.78 & 0.27 & 0.63 & 0.34 \\
\hline & 6.0 & 6.12 & 3.51 & 1.75 & 0.26 & 0.62 & 0.36 \\
\hline $504 \mathrm{~B}-78-2,62-64 \mathrm{~cm}$ & 0.2 & 5.64 & 2.99 & 1.89 & 0.30 & 0.56 & 0.25 \\
\hline$\rho=2.83 \mathrm{~g} / \mathrm{cm}^{3}$ & 0.4 & 5.73 & 3.07 & 1.86 & 0.30 & 0.57 & 0.25 \\
\hline$\phi=1.6 \%$ & 0.6 & 5.77 & 3.06 & 1.88 & 0.30 & 0.59 & 0.27 \\
\hline & 0.8 & 5.79 & 3.08 & 1.88 & 0.30 & 0.59 & 0.27 \\
\hline & 1.0 & 5.81 & 3.10 & 1.88 & 0.30 & 0.60 & 0.27 \\
\hline & 2.0 & 5.87 & 3.14 & 1.87 & 0.30 & 0.60 & 0.28 \\
\hline & 4.0 & 5.92 & 3.18 & 1.86 & 0.30 & 0.61 & 0.29 \\
\hline & 6.0 & 5.94 & 3.19 & 1.86 & 0.30 & 0.62 & 0.29 \\
\hline $504 \mathrm{~B}-79-2,33-34 \mathrm{~cm}$ & 0.2 & 5.78 & 3.02 & 1.91 & 0.31 & 0.60 & 0.26 \\
\hline$\rho=2.85 \mathrm{~g} / \mathrm{cm}^{3}$ & 0.4 & 5.81 & 3.05 & 1.90 & 0.31 & 0.61 & 0.27 \\
\hline$\phi=4.1 \%$ & 0.6 & 5.84 & 3.07 & 1.90 & 0.31 & 0.61 & 0.27 \\
\hline & 0.8 & 5.86 & 3.08 & 1.90 & 0.31 & 0.62 & 0.27 \\
\hline & 1.0 & 5.88 & 3.09 & 1.90 & 0.31 & 0.62 & 0.27 \\
\hline & 2.0 & 5.99 & 3.13 & 1.92 & 0.31 & 0.65 & 0.28 \\
\hline & 4.0 & 6.03 & 3.18 & 1.90 & 0.31 & 0.66 & 0.29 \\
\hline & 6.0 & 6.10 & 3.23 & 1.89 & 0.31 & 0.67 & 0.30 \\
\hline $504 \mathrm{~B}-80-1,9-11 \mathrm{~cm}$ & 0.2 & 4.92 & 2.69 & 1.83 & 0.29 & 0.39 & 0.20 \\
\hline$\rho=2.71 \mathrm{~g} / \mathrm{cm}^{3}$ & 0.4 & 4.96 & 2.73 & 1.81 & 0.28 & 0.40 & 0.20 \\
\hline$\phi=10.7 \%$ & 0.6 & 4.99 & 2.76 & 1.81 & 0.28 & 0.40 & 0.21 \\
\hline & 0.8 & 5.02 & 2.79 & 1.80 & 0.28 & 0.40 & 0.21 \\
\hline & 1.0 & 5.04 & 2.80 & 1.80 & 0.28 & 0.40 & 0.21 \\
\hline & 2.0 & 5.11 & 2.86 & 1.79 & 0.27 & 0.42 & 0.22 \\
\hline & 4.0 & 5.21 & 2.91 & 1.79 & 0.27 & 0.43 & 0.23 \\
\hline & 6.0 & 5.29 & 2.93 & 1.80 & 0.28 & 0.45 & 0.24 \\
\hline $504 \mathrm{~B}-82-3,74-76 \mathrm{~cm}$ & 0.2 & 6.24 & 3.29 & 1.89 & 0.31 & 0.71 & 0.32 \\
\hline$\rho=2.91 \mathrm{~g} / \mathrm{cm}^{3}$ & 0.4 & 6.32 & 3.33 & 1.90 & 0.31 & 0.73 & 0.32 \\
\hline$\phi=1.6 \%$ & 0.6 & 6.36 & 3.35 & 1.90 & 0.31 & 0.74 & 0.33 \\
\hline & 0.8 & 6.40 & 3.36 & 1.90 & 0.31 & 0.75 & 0.33 \\
\hline & 1.0 & 6.43 & 3.38 & 1.90 & 0.31 & 0.76 & 0.33 \\
\hline & 2.0 & 6.52 & 3.42 & 1.91 & 0.31 & 0.79 & 0.34 \\
\hline & 4.0 & 6.59 & 3.47 & 1.90 & 0.31 & 0.80 & 0.35 \\
\hline & 6.0 & 6.65 & 3.50 & 1.90 & 0.31 & 0.82 & 0.36 \\
\hline & 0.2 & 6.37 & 3.37 & 1.89 & 0.31 & 0.75 & 0.33 \\
\hline$\rho=2.94 \mathrm{~g} / \mathrm{cm}^{3}$ & 0.4 & 6.41 & 3.38 & 1.89 & 0.31 & 0.76 & 0.34 \\
\hline$\phi=0.3 \%$ & 0.6 & 6.43 & 3.39 & 1.89 & 0.31 & 0.76 & 0.34 \\
\hline & 0.8 & 6.44 & 3.40 & 1.90 & 0.31 & 0.77 & 0.34 \\
\hline & 1.0 & 6.46 & 3.41 & 1.90 & 0.31 & 0.77 & 0.34 \\
\hline & 2.0 & 6.51 & 3.43 & 1.90 & 0.31 & 0.78 & 0.35 \\
\hline & 4.0 & 6.55 & 3.46 & 1.89 & 0.31 & 0.79 & 0.35 \\
\hline & 6.0 & 6.58 & 3.49 & 1.89 & 0.30 & 0.80 & 0.36 \\
\hline $504 \mathrm{~B}-85-2,80-82 \mathrm{~cm}$ & 0.2 & 6.33 & 3.33 & 1.90 & 0.31 & 0.75 & 0.33 \\
\hline$\rho=2.97 \mathrm{~g} / \mathrm{cm}^{3}$ & 0.4 & 6.40 & 3.37 & 1.90 & 0.31 & 0.77 & 0.34 \\
\hline$\phi=0.5 \%$ & 0.6 & 6.43 & 3.39 & 1.90 & 0.31 & 0.77 & 0.34 \\
\hline & 0.8 & 6.46 & 3.41 & 1.89 & 0.31 & 0.78 & 0.34 \\
\hline & 1.0 & 6.48 & 3.42 & 1.89 & 0.31 & 0.78 & 0.35 \\
\hline & 2.0 & 6.56 & 3.47 & 1.89 & 0.31 & 0.80 & 0.36 \\
\hline & 4.0 & 6.62 & 3.53 & 1.88 & 0.30 & 0.81 & 0.37 \\
\hline & 6.0 & 6.66 & 3.56 & 1.87 & 0.30 & 0.82 & 0.38 \\
\hline $504 \mathrm{~B}-88-1,67-69 \mathrm{~cm}$ & 0.2 & 5.78 & 3.18 & 1.82 & 0.28 & 0.57 & 0.29 \\
\hline$\rho=2.87 \mathrm{~g} / \mathrm{cm}^{3}$ & 0.4 & 5.83 & 3.22 & 1.81 & 0.28 & 0.58 & 0.30 \\
\hline$\phi=2.8 \%$ & 0.6 & 5.86 & 3.24 & 1.81 & 0.28 & 0.58 & 0.30 \\
\hline & 0.8 & 5.88 & 3.26 & 1.80 & 0.28 & 0.59 & 0.31 \\
\hline & 1.0 & 5.90 & 3.27 & 1.80 & 0.28 & 0.59 & 0.31 \\
\hline & 2.0 & 5.99 & 3.31 & 1.81 & 0.28 & 0.61 & 0.32 \\
\hline
\end{tabular}

Table 1. (Continued).

\begin{tabular}{|c|c|c|c|c|c|c|c|}
\hline $\begin{array}{c}\text { Sample } \\
\text { (interval in cm) }\end{array}$ & $\begin{array}{c}P \\
(\mathrm{~kb})\end{array}$ & $\underset{(\mathrm{km} / \mathrm{s})}{V_{\mathrm{p}}}$ & $\begin{array}{c}V_{\mathrm{s}} \\
(\mathrm{km} / \mathrm{s})\end{array}$ & $V_{\mathrm{p}} / V_{\mathrm{s}}$ & $\sigma$ & $\begin{array}{c}K \\
(\mathrm{Mb})\end{array}$ & 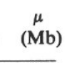 \\
\hline $\begin{array}{c}\text { 504B-88-1, 67-69 cm } \\
\text { (Cont.) }\end{array}$ & $\begin{array}{l}4.0 \\
6.0\end{array}$ & $\begin{array}{l}6.11 \\
6.22\end{array}$ & $\begin{array}{l}3.37 \\
3.42\end{array}$ & $\begin{array}{l}1.81 \\
1.82\end{array}$ & $\begin{array}{l}0.28 \\
0.28\end{array}$ & $\begin{array}{l}0.64 \\
0.67\end{array}$ & $\begin{array}{l}0.33 \\
0.34\end{array}$ \\
\hline $\begin{aligned} 504 \mathrm{~B}-89-2,30-32 \mathrm{~cm} & =2.95 \mathrm{~g} / \mathrm{cm}^{3} \\
\rho & =1.5 \%\end{aligned}$ & $\begin{array}{l}0.2 \\
0.4 \\
0.6 \\
0.8 \\
1.0 \\
2.0 \\
4.0 \\
6.0\end{array}$ & $\begin{array}{l}6.11 \\
6.18 \\
6.21 \\
6.24 \\
6.26 \\
6.33 \\
6.35 \\
6.42\end{array}$ & $\begin{array}{l}3.37 \\
3.40 \\
3.42 \\
3.43 \\
3.44 \\
3.48 \\
3.52 \\
3.56\end{array}$ & $\begin{array}{l}1.81 \\
1.82 \\
1.82 \\
1.82 \\
1.82 \\
1.82 \\
1.81 \\
1.80\end{array}$ & $\begin{array}{l}0.28 \\
0.28 \\
0.28 \\
0.28 \\
0.28 \\
0.28 \\
0.28 \\
0.28\end{array}$ & $\begin{array}{l}0.66 \\
0.67 \\
0.68 \\
0.69 \\
0.69 \\
0.71 \\
0.71 \\
0.72\end{array}$ & $\begin{array}{l}0.33 \\
0.34 \\
0.34 \\
0.35 \\
0.35 \\
0.36 \\
0.37 \\
0.38\end{array}$ \\
\hline $\begin{aligned} & 504 \mathrm{~B}-90-4,75-77 \mathrm{~cm} \\
& \rho=2.89 \mathrm{~g} / \mathrm{cm}^{3} \\
& \phi=1.4 \%\end{aligned}$ & $\begin{array}{l}0.2 \\
0.4 \\
0.6 \\
0.8 \\
1.0 \\
2.0 \\
4.0 \\
6.0\end{array}$ & $\begin{array}{l}6.35 \\
6.41 \\
6.44 \\
6.47 \\
6.50 \\
6.57 \\
6.63 \\
6.67\end{array}$ & $\begin{array}{l}3.34 \\
3.38 \\
3.41 \\
3.43 \\
3.44 \\
3.49 \\
3.54 \\
3.59\end{array}$ & $\begin{array}{l}1.90 \\
1.89 \\
1.89 \\
1.89 \\
1.89 \\
1.89 \\
1.87 \\
1.86\end{array}$ & $\begin{array}{l}0.31 \\
0.31 \\
0.31 \\
0.31 \\
0.30 \\
0.30 \\
0.30 \\
0.30\end{array}$ & $\begin{array}{l}0.73 \\
0.74 \\
0.75 \\
0.76 \\
0.76 \\
0.78 \\
0.79 \\
0.79\end{array}$ & $\begin{array}{l}0.32 \\
0.33 \\
0.34 \\
0.34 \\
0.34 \\
0.35 \\
0.36 \\
0.38\end{array}$ \\
\hline $\begin{aligned} & 504 \mathrm{~B}-91-2,15-17 \mathrm{~cm} \\
& \rho=2.95 \mathrm{~g} / \mathrm{cm}^{3} \\
& \phi=0.6 \%\end{aligned}$ & $\begin{array}{l}0.2 \\
0.4 \\
0.6 \\
0.8 \\
1.0 \\
2.0 \\
4.0 \\
6.0\end{array}$ & $\begin{array}{l}6.17 \\
6.22 \\
6.26 \\
6.28 \\
6.30 \\
6.36 \\
6.41 \\
6.44\end{array}$ & $\begin{array}{l}3.31 \\
3.34 \\
3.36 \\
3.37 \\
3.38 \\
3.41 \\
3.44 \\
3.46\end{array}$ & $\begin{array}{l}1.86 \\
1.86 \\
1.86 \\
1.86 \\
1.86 \\
1.87 \\
1.86 \\
1.86\end{array}$ & $\begin{array}{l}0.30 \\
0.30 \\
0.30 \\
0.30 \\
0.30 \\
0.30 \\
0.30 \\
0.30\end{array}$ & $\begin{array}{l}0.69 \\
0.70 \\
0.71 \\
0.72 \\
0.72 \\
0.74 \\
0.75 \\
0.76\end{array}$ & $\begin{array}{l}0.32 \\
0.33 \\
0.33 \\
0.34 \\
0.34 \\
0.34 \\
0.35 \\
0.36\end{array}$ \\
\hline $\begin{aligned} & 504 \mathrm{~B}-93-3,8-10 \mathrm{~cm} \\
& \rho=2.93 \mathrm{~g} / \mathrm{cm}^{3} \\
& \phi=1.8 \%\end{aligned}$ & $\begin{array}{l}0.2 \\
0.4 \\
0.6 \\
0.8 \\
1.0 \\
2.0 \\
4.0 \\
6.0\end{array}$ & $\begin{array}{l}6.37 \\
6.42 \\
6.46 \\
6.48 \\
6.50 \\
6.57 \\
6.64 \\
6.69\end{array}$ & $\begin{array}{l}3.42 \\
3.48 \\
3.52 \\
3.56 \\
3.59 \\
3.69 \\
3.78 \\
3.81\end{array}$ & $\begin{array}{l}1.86 \\
1.84 \\
1.83 \\
1.82 \\
1.81 \\
1.78 \\
1.76 \\
1.76\end{array}$ & $\begin{array}{l}0.30 \\
0.29 \\
0.29 \\
0.28 \\
0.28 \\
0.27 \\
0.26 \\
0.26\end{array}$ & $\begin{array}{l}0.73 \\
0.74 \\
0.74 \\
0.74 \\
0.74 \\
0.73 \\
0.74 \\
0.75\end{array}$ & $\begin{array}{l}0.34 \\
0.36 \\
0.36 \\
0.37 \\
0.38 \\
0.40 \\
0.42 \\
0.43\end{array}$ \\
\hline $\begin{aligned} 504 \mathrm{~B}-94-1,26-28 \mathrm{~cm} & =2.97 \mathrm{~g} / \mathrm{cm}^{3} \\
\phi & =0.6 \%\end{aligned}$ & $\begin{array}{l}0.2 \\
0.4 \\
0.6 \\
0.8 \\
1.0 \\
2.0 \\
4.0 \\
6.0\end{array}$ & $\begin{array}{l}6.37 \\
6.42 \\
6.46 \\
6.48 \\
6.50 \\
6.57 \\
6.64 \\
6.69\end{array}$ & $\begin{array}{l}3.42 \\
3.48 \\
3.52 \\
3.56 \\
3.59 \\
3.69 \\
3.78 \\
3.81\end{array}$ & $\begin{array}{l}1.86 \\
1.84 \\
1.83 \\
1.82 \\
1.81 \\
1.78 \\
1.76 \\
1.76\end{array}$ & $\begin{array}{l}0.30 \\
0.29 \\
0.29 \\
0.28 \\
0.28 \\
0.27 \\
0.26 \\
0.26\end{array}$ & $\begin{array}{l}0.74 \\
0.75 \\
0.75 \\
0.75 \\
0.75 \\
0.74 \\
0.75 \\
0.76\end{array}$ & $\begin{array}{l}0.35 \\
0.36 \\
0.37 \\
0.38 \\
0.38 \\
0.41 \\
0.43 \\
0.43\end{array}$ \\
\hline $\begin{aligned} & 504 \mathrm{~B}-97-1,73-75 \mathrm{~cm} \\
& \rho=2.95 \mathrm{~g} / \mathrm{cm}^{3} \\
& \phi=1.2 \%\end{aligned}$ & $\begin{array}{l}0.2 \\
0.4 \\
0.6 \\
0.8 \\
1.0 \\
2.0 \\
4.0 \\
6.0\end{array}$ & $\begin{array}{l}6.09 \\
6.14 \\
6.18 \\
6.21 \\
6.24 \\
6.32 \\
6.39 \\
6.42\end{array}$ & $\begin{array}{l}3.24 \\
3.35 \\
3.38 \\
3.40 \\
3.42 \\
3.46 \\
3.50 \\
3.54\end{array}$ & & $\begin{array}{l}0.30 \\
0.29 \\
0.29 \\
0.29 \\
0.29 \\
0.29 \\
0.28 \\
0.28\end{array}$ & $\begin{array}{l}0.68 \\
0.67 \\
0.68 \\
0.68 \\
0.69 \\
0.71 \\
0.72 \\
0.73\end{array}$ & $\begin{array}{l}0.31 \\
0.33 \\
0.34 \\
0.34 \\
0.34 \\
0.35 \\
0.36 \\
0.37\end{array}$ \\
\hline $\begin{aligned} 504 \mathrm{~B}-99-1,45-47 \mathrm{~cm} & =2.81 \mathrm{~g} / \mathrm{cm}^{3} \\
\phi & =0.3 \%\end{aligned}$ & $\begin{array}{l}0.2 \\
0.4 \\
0.6 \\
0.8 \\
1.0 \\
2.0 \\
4.0 \\
6.0\end{array}$ & $\begin{array}{l}6.34 \\
6.41 \\
6.44 \\
6.47 \\
6.49 \\
6.56 \\
6.64 \\
6.69\end{array}$ & $\begin{array}{l}3.35 \\
3.38 \\
3.40 \\
3.41 \\
3.43 \\
3.48 \\
3.53 \\
3.56\end{array}$ & & $\begin{array}{l}0.31 \\
0.31 \\
0.31 \\
0.31 \\
0.31 \\
0.30 \\
0.30 \\
0.30\end{array}$ & $\begin{array}{l}0.71 \\
0.73 \\
0.74 \\
0.74 \\
0.75 \\
0.76 \\
0.78 \\
0.79\end{array}$ & $\begin{array}{l}0.32 \\
0.32 \\
0.33 \\
0.33 \\
0.33 \\
0.34 \\
0.35 \\
0.36\end{array}$ \\
\hline $\begin{aligned} & 504 \mathrm{~B}-101-2,80-82 \mathrm{~cm} \\
& \rho=2.96 \mathrm{~g} / \mathrm{cm}^{3} \\
& \phi=0.8 \%\end{aligned}$ & $\begin{array}{l}0.2 \\
0.4 \\
0.6 \\
0.8 \\
1.0 \\
2.0 \\
4.0 \\
6.0\end{array}$ & & & & & & $\begin{array}{l}0.32 \\
0.34 \\
0.35 \\
0.36 \\
0.36 \\
0.37 \\
0.37 \\
0.38\end{array}$ \\
\hline $\begin{array}{c}\text { 04B- } 104-2,30-32 \mathrm{~cm} \\
\rho=2.94 \mathrm{~g} / \mathrm{cm}^{3} \\
\phi=0.8 \%\end{array}$ & $\begin{array}{l}0.2 \\
0.4 \\
0.6 \\
0.8 \\
1.0 \\
2.0 \\
4.0 \\
6.0\end{array}$ & $\begin{array}{l}6.24 \\
6.28 \\
6.31 \\
6.33 \\
6.34 \\
6.40 \\
6.47 \\
6.50\end{array}$ & & & $\begin{array}{l}0.33 \\
0.33 \\
0.32 \\
0.32 \\
0.32 \\
0.31 \\
0.30 \\
0.30\end{array}$ & $\begin{array}{l}0.77 \\
0.76 \\
0.76 \\
0.76 \\
0.76 \\
0.76 \\
0.77 \\
0.77\end{array}$ & $\begin{array}{l}0.28 \\
0.30 \\
0.31 \\
0.31 \\
0.32 \\
0.33 \\
0.35 \\
0.36\end{array}$ \\
\hline $\begin{aligned} 504 \mathrm{~B}-107-2,49-50 \mathrm{~cm} \\
\rho=2.94 \mathrm{~g} / \mathrm{cm}^{3} \\
\phi=1.0 \%\end{aligned}$ & $\begin{array}{l}0.2 \\
0.4 \\
0.6 \\
0.8 \\
1.0 \\
2.0 \\
4.0 \\
6.0\end{array}$ & $\begin{array}{l}5.81 \\
5.86 \\
5.89 \\
5.92 \\
5.94 \\
6.02 \\
6.12 \\
6.18\end{array}$ & $\begin{array}{l}3.13 \\
3.20 \\
3.24 \\
3.27 \\
3.28 \\
3.34 \\
3.38 \\
3.41\end{array}$ & $\begin{array}{l}1.86 \\
1.83 \\
1.82 \\
1.81 \\
1.81 \\
1.80 \\
1.81 \\
1.81\end{array}$ & $\begin{array}{l}0.30 \\
0.29 \\
0.28 \\
0.28 \\
0.28 \\
0.28 \\
0.28 \\
0.28\end{array}$ & $\begin{array}{l}0.61 \\
0.61 \\
0.61 \\
0.61 \\
0.61 \\
0.63 \\
0.66 \\
0.67\end{array}$ & $\begin{array}{l}0.29 \\
0.30 \\
0.31 \\
0.31 \\
0.32 \\
0.33 \\
0.34 \\
0.34\end{array}$ \\
\hline $\begin{aligned} & 504 \mathrm{~B}-108-1,8-10 \mathrm{~cm} \\
& \rho=2.98 \mathrm{~g} / \mathrm{cm}^{3} \\
& \phi=0.2 \%\end{aligned}$ & $\begin{array}{l}0.2 \\
0.4 \\
0.6 \\
0.8 \\
1.0\end{array}$ & $\begin{array}{l}6.26 \\
6.37 \\
6.43 \\
6.46 \\
6.49\end{array}$ & $\begin{array}{l}3.33 \\
3.44 \\
3.50 \\
3.53 \\
3.56\end{array}$ & $\begin{array}{l}1.88 \\
1.85 \\
1.84 \\
1.83 \\
1.82\end{array}$ & $\begin{array}{l}0.30 \\
0.29 \\
0.29 \\
0.29 \\
0.29\end{array}$ & $\begin{array}{l}0.73 \\
0.74 \\
0.75 \\
0.75 \\
0.75\end{array}$ & $\begin{array}{l}0.33 \\
0.35 \\
0.36 \\
0.37 \\
0.38\end{array}$ \\
\hline
\end{tabular}


Table 1. (Continued).

\begin{tabular}{|c|c|c|c|c|c|c|c|}
\hline $\begin{array}{c}\text { Sample } \\
\text { (interval in } \mathrm{cm} \text { ) }\end{array}$ & $\begin{array}{c}P \\
(\mathrm{~kb})\end{array}$ & $\begin{array}{c}V_{\mathrm{p}} \\
(\mathrm{km} / \mathrm{s})\end{array}$ & $\begin{array}{c}V_{\mathrm{s}} \\
(\mathrm{km} / \mathrm{s})\end{array}$ & $V_{\mathrm{p}} / V_{\mathrm{s}}$ & $\sigma$ & $\begin{array}{c}K \\
(\mathrm{Mb})\end{array}$ & $\stackrel{\mu}{\mu}$ \\
\hline \multirow{3}{*}{$\begin{array}{c}\text { 504B-108-1, 8-10 cm } \\
\text { (Cont.) }\end{array}$} & 2.0 & 6.58 & 3.62 & 1.82 & 0.28 & 0.77 & 0.39 \\
\hline & 4.0 & 6.65 & 3.65 & 1.82 & 0.28 & 0.79 & 0.40 \\
\hline & 6.0 & 6.67 & 3.66 & 1.82 & 0.28 & 0.80 & 0.40 \\
\hline \multirow{8}{*}{$\begin{array}{c}\text { 504B-113-1, } 12-14 \mathrm{~cm} \\
\rho=2.88 \mathrm{~g} / \mathrm{cm}^{3} \\
\phi=1.6 \%\end{array}$} & 0.2 & 5.97 & 3.18 & 1.88 & 0.30 & 0.64 & 0.29 \\
\hline & 0.4 & 6.20 & 3.23 & 1.92 & 0.31 & 0.71 & 0.30 \\
\hline & 0.6 & 6.27 & 3.27 & 1.91 & 0.31 & 0.72 & 0.31 \\
\hline & 0.8 & 6.31 & 3.31 & 1.91 & 0.31 & 0.73 & 0.32 \\
\hline & 1.0 & 6.34 & 3.33 & 1.90 & 0.31 & 0.73 & 0.32 \\
\hline & 2.0 & 6.44 & 3.38 & 1.90 & 0.31 & 0.76 & 0.33 \\
\hline & 4.0 & 6.52 & 3.44 & 1.89 & 0.31 & 0.77 & 0.34 \\
\hline & 6.0 & 6.54 & 3.50 & 1.87 & 0.30 & 0.77 & 0.36 \\
\hline \multirow{8}{*}{$\begin{aligned} 504 \mathrm{~B}-122-1,100-102 \mathrm{~cm} \\
\begin{aligned} \rho & =2.91 \mathrm{~g} / \mathrm{cm}^{3} \\
\phi & =1.2 \%\end{aligned}\end{aligned}$} & 0.2 & 6.11 & 3.30 & 1.85 & 0.29 & 0.66 & 0.32 \\
\hline & 0.4 & 6.14 & 3.34 & 1.84 & 0.29 & 0.67 & 0.33 \\
\hline & 0.6 & 6.17 & 3.37 & 1.83 & 0.29 & 0.67 & 0.33 \\
\hline & 0.8 & 6.19 & 3.39 & 1.83 & 0.29 & 0.67 & 0.33 \\
\hline & 1.0 & 6.20 & 3.40 & 1.82 & 0.28 & 0.67 & 0.34 \\
\hline & 2.0 & 6.24 & 3.44 & 1.82 & 0.28 & 0.68 & 0.35 \\
\hline & 4.0 & 6.29 & 3.46 & 1.82 & 0.28 & 0.69 & 0.35 \\
\hline & 6.0 & 6.31 & 3.46 & 1.82 & 0.28 & 0.70 & 0.35 \\
\hline & 0.2 & 6.39 & 3.22 & 1.99 & 0.33 & 0.80 & 0.30 \\
\hline$\rho=2.94 \mathrm{~g} / \mathrm{cm}^{3}$ & 0.4 & 6.43 & 3.35 & 1.92 & 0.31 & 0.78 & 0.33 \\
\hline$\phi=0.4 \%$ & 0.6 & 6.47 & 3.43 & 1.89 & 0.30 & 0.77 & 0.35 \\
\hline & 0.8 & 6.49 & 3.48 & 1.87 & 0.30 & 0.77 & 0.36 \\
\hline & 1.0 & 6.52 & 3.51 & 1.86 & 0.30 & 0.77 & 0.36 \\
\hline & 2.0 & 6.59 & 3.59 & 1.83 & 0.29 & 0.77 & 0.38 \\
\hline & 4.0 & 6.64 & 3.66 & 1.82 & 0.28 & 0.78 & 0.40 \\
\hline & 6.0 & 6.66 & 3.70 & 1.80 & 0.28 & 0.78 & 0.40 \\
\hline $504 \mathrm{~B}-128-1,25-27 \mathrm{~cm}$ & 0.2 & 6.38 & 3.27 & 1.95 & 0.32 & 0.78 & 0.32 \\
\hline$\rho=2.97 \mathrm{~g} / \mathrm{cm}^{3}$ & 0.4 & 6.43 & 3.35 & 1.92 & 0.31 & 0.78 & 0.33 \\
\hline$\phi=0.3 \%$ & 0.6 & 6.46 & 3.41 & 1.90 & 0.31 & 0.78 & 0.34 \\
\hline & 0.8 & 6.49 & 3.44 & 1.88 & 0.78 & 0.78 & 0.35 \\
\hline & 1.0 & 6.51 & 3.47 & 1.88 & 0.30 & 0.78 & 0.36 \\
\hline & 2.0 & 6.60 & 3.54 & 1.86 & 0.30 & 0.80 & 0.37 \\
\hline & 4.0 & 6.69 & 3.58 & 1.87 & 0.30 & 0.83 & 0.38 \\
\hline & 6.0 & 6.77 & 3.61 & 1.88 & 0.30 & 0.85 & 0.39 \\
\hline $504 \mathrm{~B}-129-1,27-29 \mathrm{~cm}$ & 0.2 & 6.21 & 3.23 & 1.92 & 0.31 & 0.72 & 0.31 \\
\hline$\rho=2.93 \mathrm{~g} / \mathrm{cm}^{3}$ & 0.4 & 6.30 & 3.30 & 1.91 & 0.31 & 0.74 & 0.32 \\
\hline$\phi=0.6 \%$ & 0.6 & 6.35 & 3.35 & 1.90 & 0.31 & 0.74 & 0.33 \\
\hline & 0.8 & 6.39 & 3.39 & 1.88 & 0.30 & 0.75 & 0.34 \\
\hline & 1.0 & 6.42 & 3.43 & 1.87 & 0.30 & 0.75 & 0.34 \\
\hline & 2.0 & 6.49 & 3.54 & 1.83 & 0.29 & 0.74 & 0.37 \\
\hline & 4.0 & 6.54 & 3.64 & 1.80 & 0.28 & 0.74 & 0.39 \\
\hline & 6.0 & 6.55 & 3.68 & 1.78 & 0.27 & 0.73 & 0.40 \\
\hline $504 \mathrm{~B}-130-1,75-77 \mathrm{~cm}$ & 0.2 & 6.19 & 3.29 & 1.88 & 0.30 & 0.71 & 0.32 \\
\hline$\rho=2.95 \mathrm{~g} / \mathrm{cm}^{3}$ & 0.4 & 6.23 & 3.35 & 1.86 & 0.30 & 0.70 & 0.33 \\
\hline$\phi=0.2 \%$ & 0.6 & 6.26 & 3.39 & 1.85 & 0.29 & 0.71 & 0.34 \\
\hline & 0.8 & 6.29 & 3.42 & 1.84 & 0.29 & 0.71 & 0.35 \\
\hline & 1.0 & 6.31 & 3.44 & 1.83 & 0.29 & 0.71 & 0.35 \\
\hline & 2.0 & 6.39 & 3.52 & 1.82 & 0.28 & 0.72 & 0.37 \\
\hline & 4.0 & 6.47 & 3.55 & 1.82 & 0.29 & 0.74 & 0.37 \\
\hline & 6.0 & 6.52 & 3.56 & 1.83 & 0.29 & 0.76 & 0.38 \\
\hline $504 \mathrm{~B}-131,87-89 \mathrm{~cm}$ & 0.2 & 6.31 & 3.10 & 2.04 & 0.34 & 0.80 & 0.28 \\
\hline$\rho=2.96 \mathrm{~g} / \mathrm{cm}^{3}$ & 0.4 & 6.38 & 3.27 & 1.95 & 0.32 & 0.78 & 0.32 \\
\hline$\phi=0.9 \%$ & 0.6 & 6.43 & 3.37 & 1.91 & 0.31 & 0.78 & 0.34 \\
\hline & 0.8 & 6.47 & 3.46 & 1.87 & 0.30 & 0.77 & 0.35 \\
\hline & 1.0 & 6.50 & 3.52 & 1.85 & 0.29 & 0.76 & 0.37 \\
\hline & 2.0 & 6.60 & 3.71 & 1.78 & 0.27 & 0.75 & 0.41 \\
\hline & 4.0 & 6.71 & 3.87 & 1.73 & 0.25 & 0.74 & 0.45 \\
\hline & 6.0 & 6.78 & 3.94 & 1.72 & 0.25 & 0.75 & 0.46 \\
\hline $504 \mathrm{~B}-132-2,20-22 \mathrm{~cm}$ & 0.2 & 6.07 & 3.00 & 2.02 & 0.34 & 0.72 & 0.26 \\
\hline$\rho=2.90 \mathrm{~g} / \mathrm{cm}^{3}$ & 0.4 & 6.15 & 3.05 & 2.01 & 0.34 & 0.74 & 0.27 \\
\hline$\phi=0.2 \%$ & 0.6 & 6.21 & 3.09 & 2.01 & 0.34 & 0.75 & 0.28 \\
\hline & 0.8 & 6.26 & 3.13 & 2.00 & 0.33 & 0.76 & 0.28 \\
\hline & 1.0 & 6.30 & 3.15 & 2.00 & 0.33 & 0.77 & 0.29 \\
\hline & 2.0 & 6.42 & 3.26 & 1.97 & 0.33 & 0.79 & 0.31 \\
\hline & 4.0 & 6.51 & 3.41 & 1.91 & 0.31 & 0.79 & 0.34 \\
\hline & 6.0 & 6.57 & 3.52 & 1.86 & 0.30 & 0.78 & 0.36 \\
\hline $504 \mathrm{~B}-133-2,9-11 \mathrm{~cm}$ & 0.2 & 6.44 & 3.56 & 1.81 & 0.28 & 0.73 & 0.38 \\
\hline$\rho=2.96 \mathrm{~g} / \mathrm{cm}^{3}$ & 0.4 & 6.49 & 3.66 & 1.77 & 0.27 & 0.72 & 0.40 \\
\hline$\phi=0.6 \%$ & 0.6 & 6.52 & 3.70 & 1.76 & 0.26 & 0.72 & 0.41 \\
\hline & 0.8 & 6.54 & 3.73 & 1.75 & 0.26 & 0.72 & 0.41 \\
\hline & 1.0 & 6.56 & 3.75 & 1.75 & 0.26 & 0.72 & 0.42 \\
\hline & 2.0 & 6.62 & 3.81 & 1.74 & 0.25 & 0.72 & 0.43 \\
\hline & 4.0 & 6.67 & 3.87 & 1.73 & 0.25 & 0.73 & 0.44 \\
\hline & 6.0 & 6.71 & 3.92 & 1.71 & 0.24 & 0.73 & 0.46 \\
\hline 504B-136-1, 68-70 cm & 0.2 & 6.32 & 3.51 & 1.80 & 0.28 & 0.69 & 0.36 \\
\hline$\rho=2.94 \mathrm{~g} / \mathrm{cm}^{3}$ & 0.4 & 6.34 & 3.53 & 1.79 & 0.27 & 0.69 & 0.37 \\
\hline$\phi=0.1 \%$ & 0.6 & 6.43 & 3.55 & 1.81 & 0.28 & 0.72 & 0.37 \\
\hline & 0.8 & 6.46 & 3.57 & 1.81 & 0.28 & 0.73 & 0.37 \\
\hline & 1.0 & 6.48 & 3.58 & 1.81 & 0.28 & 0.73 & 0.38 \\
\hline & 2.0 & 6.55 & 3.62 & 1.81 & 0.28 & 0.75 & 0.38 \\
\hline & 4.0 & 6.59 & 3.69 & 1.79 & 0.27 & 0.75 & 0.40 \\
\hline & 6.0 & 6.62 & 3.73 & 1.77 & 0.27 & 0.75 & 0.41 \\
\hline
\end{tabular}

Table 2. Average velocities at $1 \mathrm{~kb}\left(V_{\mathrm{p}}\right.$ and $V_{\mathrm{S}}$ ), densities $(\rho)$, and porosities $(\phi)$ of Hole 504B basalts.

\begin{tabular}{ccccc}
\hline & $\begin{array}{c}\mathrm{V}_{\mathrm{p}} \\
(\mathrm{km} / \mathrm{s})\end{array}$ & $\begin{array}{c}\mathrm{V}_{\mathrm{s}} \\
(\mathrm{km} / \mathrm{s})\end{array}$ & $\begin{array}{c}\rho \\
\left(\mathrm{g} / \mathrm{cm}^{3}\right)\end{array}$ & $\begin{array}{c}\phi \\
(\%)\end{array}$ \\
\hline $3-8$ & 6.26 & 3.32 & 2.86 & 1.7 \\
$9-15$ & 6.12 & 3.32 & 2.88 & 2.1 \\
$16-23$ & 6.14 & 3.31 & 2.86 & 1.7 \\
$24-33$ & 6.13 & 3.33 & 2.87 & 2.2 \\
$34-45$ & 5.85 & 3.15 & 2.77 & 5.8 \\
$45-62$ & 6.43 & 3.47 & 2.93 & 1.3 \\
$63-71$ & 5.98 & 3.18 & 2.86 & 2.4 \\
$72-77$ & 6.07 & 3.24 & 2.88 & 1.2 \\
$78-83$ & 5.79 & 3.09 & 2.83 & 4.5 \\
$84-89$ & 6.28 & 3.39 & 2.93 & 1.3 \\
$90-95$ & 6.45 & 3.50 & 2.94 & 1.1 \\
$96-105$ & 6.34 & 3.41 & 2.92 & 0.8 \\
$106-125$ & 6.24 & 3.40 & 2.93 & 1.0 \\
$125-130$ & 6.44 & 3.46 & 2.95 & 0.4 \\
$131-136$ & 6.46 & 3.50 & 2.94 & 0.5 \\
\hline
\end{tabular}

